\title{
Balkanologie
}

Balkanologie Revue d'études pluridisciplinaires

Vol. VII, n 2 | 2003

Volume VII Numéro 2

\section{Entre ruralité et urbanité : les institutions de voisinage en Transylvanie}

Anne Schiltz

\section{(2) OpenEdition}

\section{Journals}

Édition électronique

URL : http://journals.openedition.org/balkanologie/498

DOI : 10.4000/balkanologie.498

ISSN : 1965-0582

Éditeur

Association française d'études sur les Balkans (Afebalk)

Édition imprimée

Date de publication : 1 décembre 2003

Pagination : 99-126

ISSN : 1279-7952

\section{Référence électronique}

Anne Schiltz, "Entre ruralité et urbanité : les institutions de voisinage en Transylvanie », Balkanologie

[En ligne], Vol. VII, n² 2 2003, mis en ligne le 19 février 2009, consulté le 17 décembre 2020. URL http://journals.openedition.org/balkanologie/498 ; DOI : https://doi.org/10.4000/balkanologie.498

\section{(c) Tous droits réservés}




\section{DOSSIER}

\section{URBANITÉ ET RURALITÉ}




\title{
ENTRE RURALITÉ ET URBANITÉ : LES INSTITUTIONS DE VOISINAGE EN TRANSYLVANIE
}

\author{
Anne Schiltz*
}

En Transylvanie, la Nachbarschaft, terme allemand dont les traductions roumaine et hongroise sont respectivement vecinătate et szomszédság et qu'on pourrait traduire en français par "l'institution du voisinage", est une institution spécifique aux villages saxons de Transylvanie qui se révèle comme un exemple précis de ce qu'on pourrait appeler de façon générale "les relations de voisinage". Les Saxons se sont installés en Transylvanie au cours du XII ${ }^{\mathrm{e}}$ siècle. La Nachbarschaft a évolué dans le paysage transylvain, caractérisée par une dynamique d'influences mutuelles roumaines, tsiganes, hongroises et saxonnes.

La pratique institutionnalisée de relations de voisinage et par extension de relations d'entraide autres que de voisinage s'est transmise sur un axe diachronique et synchronique, entre générations ainsi qu'entre groupes définis comme groupes "nationaux" ou encore "ethniques" en Transylvanie ${ }^{1}$.

À partir de l'exemple des institutions de voisinages transylvaines, nous proposons de débattre de l'application pertinente des catégories ruralité/urbanité à cette institution. Est-ce que le mode de fonctionnement de l'institution rurale de voisinage est en rupture par rapport à l'expression urbaine de cette organisation sociale ? Et si oui, en quoi l'emplacement en milieu rural ou urbain viendrait-elle expliquer cette rupture?

Au lieu de séparer deux formes d'être en voisinage, nous proposerons d'articuler les relations de voisinage en réseaux pour donner à voir plutôt des institutions hybrides que des voisinages urbains et ruraux. Cette articulation en

\footnotetext{
- Doctorante. Centre de Recherche en Ethnologie Européenne (Université Libre de Bruxelles, Institut de Sociologie), Bruxelles.

${ }^{1}$ Le terme de nation rejoint ici l'acception allemande du terme "nation" qui se définit à travers le lien du "sang" et de la "communauté culturelle", Kulturgemeinschaft et non par rapport à l'État-Nation, il ne s'agit pas de la citoyenneté ; les termes "nation" et "ethnie" sont donc utilisés ici comme synonymes.
} 
réseaux permettra de comprendre tout d'abord l'institution de voisinage en tant que processus dynamique jonglant entre traits typiquement "ruraux" ou "urbains" ainsi que de poser la question de l'efficacité de la catégorisation ethnique en Transylvanie.

\section{RELATIONS DE VOISINAGE ET IDÉAL COMMUNAUTAIRE}

Le dictionnaire des frères Grimm indique que le terme allemand de Nachbar, "voisin" se compose de nach, nahe qui veut dire "près de" et bur qui signifie Bauer, c'est-à-dire "paysan"2.

Dans la littérature sociologique, l'idéal communautaire se traduit à travers des relations qui sont par excellence des relations de voisinage, de neighbourhood. Le critère spatial, la "localité" joue un rôle déterminant dans l'articulation de la dichotomie classique entre ville et village. Bien que Tönnies, un père fondateur de la sociologie rurale, ait été remis en question, la dichotomie entre Gemeinschaft et Gesellschaft a eu, sans aucun doute, une large influence sur les monographies de communautés rurales. La Gemeinschaft, telle que définie par Tönnies, présuppose des relations humaines intenses, développées à travers la parenté, le voisinage et l'amitié, le lien à un espace partagé et l'action commune dans la coordination et la coopération en vue du bien commun. Par opposition, la Gesellschaft consiste en relations impersonnelles et basées sur l'échange formel et le contrat. Cette "société" ou "association" prend forme lorsque les populations s'industrialisent 3 . L'analyse du passage ou de la "transition" vers l'économie de marché est à la base du corpus d'analyses considérable qui fonde les diverses "théories de la modernité".

Parmi ces dernières, Reissman qualifie les analyses se basant sur ce type de dichotomies de "so-called theories of contrast " en précisant que leur valeur heuristique n'a jamais été prouvée.

Cependant, ni Weber, ni Durkheim ne qualifiaient ces tendances comme archétypes d'ères historiques différentes qui coïncideraient, mais plutôt comme tendances contrastées au sein d'une société à un moment ou un autre. Durkheim parle de différences dans les relations qu'entretiennent les individus avec la société, il situe ces différences dans les processus sociaux. Anthony P. Cohen suggère que la complémentarité des deux modes d'être en collectivité fût par la suite largement négligée, et ce, notamment, dans la tra-

\footnotetext{
2 Schubert (Hans-Achim), Nachbarschaft und Modernisierung. Eine historische Soziologie traditionaler Lokalgruppen am Beispiel Siebenbürgens, Kôln : Bôhlau Verlag (Studia Transylvanica, 3), 1980, p. 17.
}

${ }^{3}$ Harper (Sarah), "The British rural community : an overview of perspectives ", Journal of Rural Studies, 5 (2), 1989, pp. 162-163. 
dition de l'école de Chicago4. Celle-ci aurait utilisé la dichotomie proposée par Durkheim comme paradigme fondant leurs propres distinctions entre sociétés rurales et urbaines, qualifiées respectivement comme simples et complexes5.

Pour Henri Mendras il n'y a de paysans que depuis qu'il y a des villes :

l'opposition entre villes et campagnes s'est affirmée au XIXe siècle avec l'expansion urbaine et le développement industriel. Aujourd'hui, après sa troisième révolution, le secteur agricole a perdu les trois quarts de ses paysans pour ne plus conserver que des agriculteurs. La campagne redevient un lieu de vie pour toutes les catégories de la population. (...) il faut donc réviser complètement notre image des campagnes et du milieu rural. ${ }^{6}$

Tel que Mendras, Georges Duby parle de la France et plus précisément de la Provence du XIV e siècle lorsqu'il nous dit que " dans ce pays, les phénomènes urbains et ruraux s'interpénètrent, ce qui amène l'historien de la campagne à formuler de manière particulière ses interrogations " 7 .

En effet, la distinction a priori de rapports sociaux sur la base de leur emplacement spatial est un exercice particulièrement stérile.

Pour aller au-delà de la dichotomie analytique ville/village, le concept de continuum rural-urbain a fait surface au cours des années soixante ${ }^{8}$. Proposer d'atténuer la dichotomie en la rattachant à une échelle linéaire posera d'autres difficultés. Dans son article intitulé " The Rural-Urban Continuum ", R.E. Pahl met en évidence le risque de faire pis que mieux en acceptant trop rapidement un faux continuum rural-urbain pour aller au-delà de la dichotomie ville/village. Il parle de l'existence de plusieurs séries de continua mais également de discontinuités abruptes, particulièrement dans la confrontation entre le local et le national :

wether we call the processes acting on local community "urbanization”, “differentiation", "modernization", "mass-society", or whatever, it is clear it is not so much communities that are acted upon as groups and individuals at particular places in the social structure. Any attempt to tie particular patterns of social relationships to specific geographical milieux is a singularly fruitless exercise. 9

${ }^{4}$ Les auteurs pionniers étant Robert Park, Ernest Burgess (entre-deux-guerres) et plus tard Louis Wirth.

5 Cohen (Anthony P.), The symbolic construction of community, London : Tavistock Publications, 1985, pp. 24-28.

${ }^{6}$ Mendras (Henri), Les sociétés paysannes, Paris : Gallimard, 1976, p. 225.

7 Duby (Georges), Le dimanche de Bouvines, Paris : Gallimard, 1973, p. 253.

${ }^{8}$ Dewey (Richard), "The Rural-Urban Continuum : Real but Relatively unimportant ", American Journal of Sociology, 66 (1), 1960 ; Benet (Francisco), " Sociology Uncertain : The Ideology of the Rural-Urban Continuum ", Comparative Studies in Society and History, 6 (1), octobre 1963 ; Hauser (P. M.), 1965, "Observations on the urban-folk and Urban-rural Dichotomies as forms of Western Ethnocentrism ", Studies of Urbanization, 1965 (cités in Pahl (Raymond E.), "The rural-urban continuum ", Sociologia Ruralis, 6 (3-4), 1966).

9 Pahl (Raymond E.), art. cit., p. 85. 
L'auteur propose d'approcher le continuum rural-urbain en tant que processus et dans la diachronie plutôt que dans l'espace à proprement parler. En d'autres termes, ce n'est pas parce qu'on se trouve dans un contexte urbain que les relations entre individus sont exclusivement formelles, caractérisées par l'anonymat d'un côté et la spécialisation de l'autre. Pahl fait référence aux villages urbains, "métropolitains" ou aux groupes informels, créés par exemple sur le lieu de travail.

La dichotomie analytique village/ville, mise en question par tant de chercheurs en sciences sociales pour l'Europe occidentale, ne saurait davantage être valable a priori pour l'Europe orientale. L'importance de la paysannerie, notamment en Roumanie, et le rôle qu'elle a joué en tant qu'objet d'étude en sciences sociales repose la question de la pertinence de l'application des catégories de ruralité et surtout d'urbanité. Nous faisons référence ici aux recherches menées sous la direction de Dimitrie Gusti en Roumanie, et à son programme des monographies villageoises, qui a pu aboutir à une riche analyse comparative et typologique. Et Henri Stahl, sur la base de recherches empiriques des réalités sociales, très approfondies, a su instituer le "village" en tant qu'unité d'analyse à valeur explicative, en mettant en évidence la solidarité pénale, fiscale, sociale et économique des anciennes communautés villageoises roumaines ${ }^{10}$.

Sans pouvoir ici approfondir les vastes sujets tels le phénomène du second servage, les relations entre villages et villes en Transylvanie et finalement les effets d'un régime totalitaire s'appuyant notamment sur la paysannerie, nous proposerons une analyse à travers l'exemple concret de l'institution de voisinage qui, traversant toutes ces étapes nommées "périodes", a su être réitérée en réalité sociale jusqu'à nos jours.

Plutôt qu'a priori urbains ou ruraux, ces groupes d'entraide ou réseaux d'entraide, insérés activement dans d'autres réseaux de relations, répondent aux contextes divers par une dynamique d'adaptation à travers un processus de réinvention permanente.

Mendras, Pahl, Granovetter, Wellman et bien d'autres proposent le paradigme de réseau pour aller au-delà de l'analyse des catégories pour privilégier celle des relations. En d'autres termes, le monde ne serait pas composé de groupes, mais de réseaux. Cette approche a le mérite de prendre en considération aussi bien les relations qui ne donnent pas lieu à la formation de groupes que les relations qui sont suffisamment serrées et récurrentes pour qu'on les nomme "groupes". "Indeed, they [structural analysts] caution that descriptions based on bounded groups oversimplify complex social structures, treating them as organizational trees, when it is the network members' 
crosscutting memberships in multiple social circles that weave together social systems (an argument dating back to Simmel). $n^{11}$

\section{L'INSTITUTION DE VOISINAGE : UNE BRÈVE ESQUISSE}

L'institution de voisinage transylvaine est une organisation sociale d'entraide qui se définit autant à travers la structure des relations entre membres, régie par des normes écrites et orales, qu'à travers sa relation à d'autres réseaux, divers, locaux et supra-locaux. La pratique de l'institution du voisinage s'étend sur des siècles, la structure interne et la qualité des relations entre membres ainsi que le rôle revêtu par cette organisation parmi d'autres ont bel et bien changé au cours du temps.

Une première brève présentation de ce mode d'organisation s'avère utile à ce stade, les détails constitutifs feront intégralement partie de l'analyse proposée par la suite.

L'objectif de l'institution du voisinage est l'entraide, et la réalisation de l'entraide passe par une fixation, écrite ou orale, de règles appelées statuts qui se traduisent par des amendes en cas de non-respect.

L'appartenance à l'institution de voisinage, se définissait, du côté saxon, sur la base territoriale alors que ce critère ne joue plus un rôle exclusif aujourd'hui et ce d'autant plus quand il s'agit de voisinages non-saxons.

L'appartenance à une institution de voisinage se définit à travers un ensemble de critères possibles mais l'appartenance à un des groupes "nationaux" se révèle être déterminant et efficace. I'institution du voisinage, en tant que pratique s'est bien répandue en Transylvanie et ce à travers les trames de relations existantes entre les différents groupes mais en ne franchissant guère les frontières de celles-ci. Ceci revient à dire que les membres du voisinage saxon sont, à quelques exceptions près, saxons et que le voisinage en question est nommé "voisinage saxon". Et il en va de même pour les voisinages roumains, tsiganes et hongrois. Le critère territorial est valable dans le voisinage saxon et les membres de ce voisinage sont véritablement voisins entre eux. Cependant, les voisins roumains ou tsiganes n'entreront pas en tant que membres dans l'institution de voisinage saxonne de la rue en question.

Les fonctions auxquelles répond l'institution de voisinage ont bel et bien changé au cours des siècles, alors que la forme, le mode d'organisation ou encore le patron présentent une continuité étonnante.

\footnotetext{
${ }^{11}$ Wellman (Barry), "Structural Analysis : from method and metaphor to theory and substance ", in Wellman (Barry), Berkowitz (Stephen D.), eds., Social structures : a network approach, New York : Cambridge University Press, 1988, p. 37.
} 
Chaque institution est dirigée par un "père de voisinage" qui est élu pour une période variable (il s'agit souvent de deux ans) avec néanmoins la possibilité de reconduire le mandat indéfiniment. Tous les membres masculins sont théoriquement susceptibles de devenir au moins une fois père de voisinage au cours de leur vie. En principe, on se réfère à l'ordre d'inscription des membres pour désigner la succession proposée. Cette proposition est sanctionnée ou non par l'ensemble des membres masculins qui se réunissent au moins une fois par an, au moment du Richttag ou riștog ou ședința anuală de l'institution. Lors de cette réunion annuelle - obligatoire pour tous les membres sous peine d'amende - le point est fait sur les activités de l'institution au cours de l'année écoulée et sur les décisions à prendre en commun pour l'avenir. Le trésorier présente son rapport sur les entrées et sorties dans la caisse du voisinage qui est alimentée par les amendes payées, les taxes d'inscription des nouveaux membres, les cotisations exceptionnelles décidées en commun et éventuellement par la vente d'objets appartenant à l'institution. En effet, le voisinage dispose très souvent de tables, de vaisselle, de chaises et de marmites qu'il prête à ses membres lorsque ceux-ci doivent organiser une grande fête tel un mariage, un enterrement ou encore un baptême.

La communication des messages suit un cheminement bien précis. Les informations sont émises par et à travers la personne du père de voisinage ou de son assistant appelé "jeune ancien" (Jungälteste) ou "petit père de voisinage" (tatal mic). L'information est transmise soit à son voisin physique soit à un Zehntmann ou sectorișt, un responsable d'un quartier, qui à son tour communique le message. Le message bénéficie souvent d'un support matériel, un morceau de bois, en forme de cour ou de pique, qui passe de main en main pour faire le tour de toutes les familles-membres. À cette "tablette de voisinage" peut être attaché un bout de papier avec un message écrit, mais elle peut également fonctionner comme support mnémonique et symbolique à l'information transmise oralement.

Les messages transmis concernent le lieu et la date de réunion et, plus particulièrement, l'annonce des décès au sein de cette communauté associative.

La prise en charge de l'enterrement est actuellement au centre de la majorité des communautés de voisinages. Chaque décès relance l'organisation du voisinage et exige des familles-membres une participation physique et très souvent financière. En effet, il n'est pas rare que le voisinage offre une aide financière ou le cercueil à la famille du défunt. Des membres masculins creusent la tombe destinée à accueillir un de leurs "voisins" alors que d'autres se chargent du transport du défunt jusqu'à sa demière demeure.

Ainsi, un décès active le lien entre membres et collectivité ; la prise en charge de l'enterrement mobilise l'ensemble des membres. 


\section{LA NACHBARSCHAFT DANS LA DIACHRONIE : INSTITUTIONS SAXONNES DE VOISINAGE, RURALES ET URBAINES}

Le débat sur l'origine de l'institution du voisinage pose la question de l'origine rurale ou urbaine de cette institution.

En effet, il existe deux hypothèses concernant l'origine de la Nachbarschaft. La première avance que des colons appelés Saxons ont amené en terre transylvaine cette pratique d'organisation sociale au cours du XII siècle. Selon cette hypothèse, la pratique du voisinage trouverait son origine dans les anciennes Dorfgenossenschaften ou communautés villageoises de la région franco-mosellane et s'inscrirait alors dans la longue durée selon l'acception braudélienne du terme. Selon l'autre hypothèse, la Nachbarschaft se serait organisée en se frottant aux corporations médiévales saxonnes en Transylvanie. Il est reconnu que les institutions de voisinage se confondaient parfois avec les corporations. Dans ce cas, l'institution était composée des maîtres d'une profession artisanale, habitant une rue de la ville et prenant en charge l'éventail des fonctions d'une institution de voisinage.

Originairement, et il s'agirait là d'un trait commun à la Dorfgenossenschaft franco-mosellane aussi bien qu'à l'institution du voisinage, le soin des puits communs ainsi que la digne organisation de funérailles a été au centre des préoccupations de ces organisations. En ville, des patrouilles étaient organisées au sein du voisinage pour veiller, la nuit, à la sécurité du quartier.

Stefan Ludwig Roth, le grand écrivain saxon, donnait une définition, désormais classique, de la Nachbarschaft :

die aus einem Brunnen tranken, Brot aus einem Ofen aßen, die die Nachthut für einander hielten, die sich ihre Wohnhäuser aus gemeinschaftlicher Kraft aufrichteten, in Krankheits-und Unglücksfallen den Willen der Anverwandten hatten, die endlich einmal alle auf derselben Totenbank ruhten, die sich einander ihre Gräber gruben, eigenhändig ihren Toten auf den Gottesacker trugen und die letzte traurige Ehre der Leichenbegleitung als eine Gemeinsamkeit erwiesen, beim Tränenbrote der Verschiedenen Verdienste rühmten und aus nachbarlichem Vermogen und Beruf für Witwen und Waisen sorgten -diese brüderliche Gesellschaft, durch Örtlichkeit bezeichnet, nannte sich die "Nahen", die "Nachbarschaft"

12 Cité par Weber (Georg), "Zum Struktur-und Funktionswandel der siebenbürgisch-sächsischen Nachbarschaft in Rumänien und West Deutschland ", Ethnologia Europaea, 12 (2), 1981, p. 149.

"Ceux quí boivent du puits commun, mangent le pain cuit dans le four commun, ceux qui veillaient la nuit les uns pour les autres, qui construisaient ensemble leurs maisons, qui prenaient en charge les familles frappées par la maladie ou par un accident, ceux qui finalement reposaient tous sur un même banc funéraire et qui se creusaient leurs tombes les uns les autres, et portaient à mains nues leur défunt jusque dans la tombe en lui rendant un dernier honneur lors du discours d'adieu, c'est le voisinage qui pratiquement et matériellement prend en charge les orphelins et les veuves -cette société fraternelle, caractérisée par un lien à l'espace commun, se nommait les "proches", le "voisinage". " 


\section{Dimension et spécialisation de la Nachbarschaft}

En ce qui concerne le déploiement de la pratique en Transylvanie, H.-A. Schubert considère que l'organisation de la Nachbarschaft rurale serait comparable à la Nachbarschaft urbaine. Cependant, en ville, l'existence des Zehntschaften ${ }^{13}$ et des Brunnennachbarschaften ${ }^{14}$ comme en général les liens avec l'administration locale sont bien plus accentués et formalisés qu'en milieu rural. La taille des institutions urbaines varie en fonction des devoirs à remplir.

Ainsi le nombre de membres des voisinages-corporations variait en fonction de leurs moyens de production et du marché d'écoulement ; la Brunnennachbarschaft comptait plus ou moins de membres selon le volume d'eau du puits, du système de récupération d'eau ainsi que des moyens de transport.

La taille des unités responsables de la sécurité dépendait des armes et d'autres objets de protection, comme également de la nature et du nombre des ennemis désignés. En milieu urbain, on assiste à une certaine spécialisation des fonctions des différentes institutions de voisinage.

Alors que les voisinages urbains pouvaient réunir jusqu'à 120 familles, réparties en sous-unités, en milieu rural, la Nachbarschaft comptait autour de 20 à 30 familles-membres. Les réseaux de contacts formels et informels restent ici largement identiques. La taille de leurs groupes de travail restait relativement constante. En cas de travaux concernant le village entier, les différents voisinages s'entraidaient.

\section{L'insertion de l'institution du voisinage dans une hiérarchie de réseaux}

Au cours de l'histoire, ces différentes institutions de voisinage saxonnes ont été liées plus ou moins étroitement à des instances politico-religieuses supérieures. Lorsque les groupes locaux perdaient leurs fonctions au niveau politique et militaire au profit de collectifs plus larges et plus spécialisés tels les administrations communales et les associations civiles de défense, les réseaux de voisinage informels, d'amitiés et de parenté se détachaient progressivement des systèmes d'interaction organisés.

En effet, les Saxons ont joui longtemps d'une relative autonomie politique et juridique ${ }^{15}$. La Nachbarschaft, en tant qu'organisation sociale s'inscrit dans l'influence de ces réseaux structurés sur base coopérative. Les réseaux de fonc-

\footnotetext{
13 Il s'agit de sous-unités, responsables de fonctions spécifiques.

${ }^{14}$ Les voisinages du puits qui, comme le terme l'indique, s'occupaient prioritairement de l'hygiène des puits.
}

15 Appelés par le roi hongrois Geisa Il pour coloniser et protéger la frontière de l'empire, menacée de l'intérieur par la noblesse et de l'extérieur par divers envahisseurs. La " lettre dorée Andreanum " renouvelée par les rois hongrois depuis Andreas Il en 1224 leur confère le libre droit à la propriété foncière, le libre 
tionnement et d'organisation se basaient sur la coopération d'individus relativement égaux et libres qui ne connaissaient pas le régime féodal et étaient responsables de leur sort.

Au cours du temps, les administrations communales transylvaines ont régulièrement essayé de se lier aux institutions de voisinages à travers l'assignation de travaux publics de tout genre.

Alors que les voisinages avaient longtemps établi leurs propres statuts, ceux-ci leur furent plus tard octroyés en partie par le magistrat en collaboration avec le père de voisinage.

Des fonctions de défense et de surveillance aux portes des villes leur furent attribuées, celles, entre autres, d'empêcher l'achat de maisons au sein des villes saxonnes par des ressortissants de nations étrangères. En effet, alors qu'un protocole de 1768 témoigne de l'interdiction du droit à l'habitat des Roumains (installés en grand nombre dans la périphérie) au centre de la ville de Sibiu, les autorités de Vienne ordonnent l'insertion de tous les propriétaires voisins dans les institutions de voisinages, sans égard distinction de nationalité ${ }^{16}$.

Depuis le XVlle siècle, "l'Eglise transylvaine de nationalité saxonne", Eglise luthérienne, a su se servir avec succès de la structure des relations de voisinages institutionnalisées en tant qu'instrument d'une éducation chrétienne, de contrôle ecclésiastique et de sanction.

En milieu rural, de façon générale, les Nachbarschaften ont su garder une autonomie certaine par rapport aux organes d'administrations communales. Par contre, ce sont ici les instances religieuses à travers le consistoire de la commune, du district ou du pays qui dictent la rédaction des statuts de voisinages.

L'autonomie politique des Saxons ne prit véritablement fin qu'en 1876 après l'intégration de la Transylvanie en 1867 à la partie hongroise de la double monarchie.

Les conséquences de cette intégration politique pour les institutions des voisinages se concrétisèrent en 1891 à travers un décret émanant du ministère hongrois et enlevant aux voisinages leurs fonctions juridico-publiques en tant qu'organes de la commune ou de la société civile urbaine.

En milieu urbain, l'institution du voisinage disparaît progressivement au début du XX $\mathrm{XX}^{\mathrm{e}}$ siècle en laissant aux organisations spécialisées le soin de prendre en charge les différents aspects de la vie publique des citoyens. Ce n'est qu'après la Première Guerre que les institutions de voisinage furent réanimées

choix des prêtres, le droit d'usage commun des forêts et de l'eau, la liberté douanière pour leurs commerçants et leurs marchés ainsi que la prise en charge de leur propre protection ; ces libertés constituaient la base de leur autonomie.

${ }^{16}$ Schenk (Annemie), " Interethnischer Austausch zwischen Siebenbürger Sachsen und Rumanen am Beispiel der Institution der Nachbarschaft n, in Tolksdorf (Ulrich), Hrsg., Sonderausdruck aus : Jahrbuch für ostdeutsche Volkskunde, Vol. 30, Marburg : Deutsche Gesellschaft für Volkskunde, 1987, p. 166. 
en tant qu'" outils de protection du peuple ", avec l'accent mis de plus en plus sur l'appartenance ethnique. C'est seulement au début des années 1930, suite à l'émergence de constellations politiques nouvelles, que les Nachbarschaften ont perdu leur attachement exclusif à l'Eglise luthérienne pour devenir des völkische Nachbarschaften, des "voisinages du peuple", et donc à caractère ethnique. Désormais les institutions de voisinage furent également accessibles aux quelques Saxons catholiques ${ }^{17}$.

Après la Deuxième Guerre et les années de déportation d'une grande partie de la population saxonne en Union soviétique, les Saxons qui revinrent s'installer dans leur village natal commencèrent à s'organiser de nouveau en voisinages. $90 \%$ des propriétés foncières saxonnes devenaient propriété étatique et les paysans perdaient, en plus de leurs terres, également leurs maisons et leurs outils de travail. Ceux-ci étaient redistribués à des populations paysannes roumaines ${ }^{18}$ de régions agricoles plus pauvres ainsi qu'à une partie des Tsiganes du village et de la région ${ }^{19}$. Étant donné la diminution de la population saxonne suite à la déportation ainsi que la délocalisation des ménages, due aux changements de propriètés, les Saxons ne respectaient plus le critère territorial, du moins jusqu'à la réintégration de leurs fermes en tant que propriétaires dans les années $190^{20}$. Le recrutement des membres se fait toujours exclusivement sur base du critère ethnique. Selon Georg Weber, sociologue saxon, les fonctions de la Nachbarschaft se seraient réduites après la Deuxième Guerre. Et au rôle primordial joué au sein de la communauté, ne répondrait désormais plus qu'une unité sociale relativement formelle et liée directement à la communauté évangélique-luthérienne ${ }^{21}$.

La Nachbarschaft est réarticulée en milieu rural et fonctionnera durant les années du régime socialiste pour ne laisser que des traces dans la plupart des villages après $199^{\circ}$, l'année qui connaît une émigration massive des Saxons, vers l'Allemagne surtout. Cependant, à Sighișoara, ville de taille moyenne, les Saxons continuent à fonctionner en voisinages, voisinages féminins et mascu-

${ }^{17}$ Schurn (Wilhelm), Die Nachbarschaften der Deutschen in Rumänien, Hermannstadt : Krafft \& Drotleff, 1936.

${ }^{18}$ Dans le village de Mălâncrav ainsi que dans les villages voisins, ces populations furent appelees "colonistes" et vinrent, pour Māläncrav, de la région du Somes, près de Cluj. Ils venaient suite à des appels des maires des villages d'accueil et des accords passés avec les populations accueillies ; il est difficile de trouver de la documentation précise à ce sujet. Après 1956, une partie de ces gens quittaient les villages d'accueil, d'autres furent encouragés, à travers des subventions étatiques, à se construire leurs propres maisons dans ces villages saxons ; dans ces villages transylvains, les nues entières construites fin des années 1950 par les "colonistes" gardent leur dénomination de "rue des colonistes". À Mălâncrav, les Rournains colonistes ont fondé une institution de voisinage.

19 Gündisch (Konrad), Siebenbürgen und die Siebenbürger Sachsen, München : Langen Müller, 1998, p. 224.

${ }^{20}$ En 1956, la majorité des maisons et des jardins furent rendus aux anciens propriétaires.

${ }^{21}$ Weber (Georg), art. cit., p. 146. 
lins. La prise en charge des enterrements, tâche importante pour toute institution de voisinage, devient ici, et plus particulièrement en ce qui concerne la Nachbarschaft masculine, la fonction unique de cette organisation. Une coopération directe lie les institutions saxonnes à l'Eglise luthérienne, en milieu rural ainsi qu'en milieu urbain en ce qui concerne la composante féminine des voisinages à Sighișoara.

L'institution du voisinage est loin de disparaitre, en milieu rural comme en milieu urbain, les voisinages roumains, hongrois et tsiganes sont bien vivants alors que les voisinages saxons sont recrées par les Saxons émigrés en Allemagne et en Autriche.

L'évolution de la Nachbarschaft met en évidence l'articulation complexe des répercussions des contextes politiques et historiques ainsi que du développement propre au paysage social, rural et urbain.

\section{Discontinuité des institutions de voisinages et continuité de l'institution en tant que pratique transylvaine}

Les institutions de voisinage roumaines et hongroises ne sont pas des créations récentes. Pour Sighișoara, des archives communales évoquent l'existence d'institutions de voisinage dès l'année 1767,1768 et $1788^{22}$. Les premières traces écrites de voisinage hongrois datent de 1636 et se réfèrent à celui du quartier Bartolomeu à Brașov. À Sighișoara encore, suite à l'augmentation continue de la population hongroise après le traité de 1867 , les voisinages hongrois s'affirment et sont actuellement au nombre de cinq ${ }^{23}$. Il n'existe pour ainsi dire pas de documentation ni de recherches approfondies sur la version tsigane de cette institution. Pourtant, ces derniers ont également adopté ce mode d'entraide institutionnalisé, aussi bien en milieu rural qu'en milieu urbain. À Sighișoara toujours, selon le président du parti des Tsiganes de Sighișoara, il existerait six de ces voisinages. Dans les villages, tous les Tsiganes se réunissent généralement en un grand voisinage sans tenir forcément compte du critère territorial.

Cette institution s'est frottée au temps, s'est transmise et bien qu'ayant gardé la dénomination de Nachbarschaft, Vecinătate ou Szomszédság ainsi que le modèle élémentaire, elle a su être adaptée en faisant preuve d'une grande flexibilité. Sa pratique n'a été continue ni dans l'espace ni dans le temps sans s'effacer pour autant de la mémoire de ceux qui, dans des contextes divers, la font revivre.

Quelques exemples : les institutions roumaines de voisinage à Slimnic (village à proximité de la ville de Sibiu) créées sous l'influence de l'adminis-

\footnotetext{
${ }^{22}$ Schenk (Annemie), art. cit., p. 168.

${ }^{23}$ Pozsony (Ferenc), "Vecinitatile din Transilvania ", in Mihailescu (Vintila) et al., ed., Vecini si Vecinatati in Transilvania, Bucarest : Paideia, 2002, p. 39.
} 
tration communale et attestées au XVIII ${ }^{\mathrm{e}}$ et au début du XIX ${ }^{\mathrm{e}}$ siècle, disparaissent pour être réinventées au cours du $\mathrm{XX}^{\mathrm{e}}$ siècle. Les voisinages saxons de Sibiu prennent fin suite à un conflit avec un membre "de nationalité étrangère", un directeur des finances hongrois ; conflit qui consomme définitivement le décret émanant du ministère hongrois de 1891 qui enlève aux institutions de voisinage leurs fonctions juridico-publiques en tant qu'organes de la commune ou de la société civile urbaine ${ }^{24}$. En milieu rural, les institutions restent plus indépendantes par rapport aux administrations communales, le décret de 1891 ne les touche guère et elles continuent à fonctionner en tant qu'organisations nationales et/ou religieuses (en ce qui concerne les voisinages saxons) répondant à un large éventail de besoins d'entraide, formelle et informelle.

Lors de ses visites des paroisses dans la deuxième moitié du XIX ${ }^{\mathrm{e}}$ siècle, l'évêque protestant $G$. $D$. Teutsch réanima les institutions saxonnes de voisinage si celles-ci n'existaient pas ou plus en tant qu' "institution ecclésiastique du peuple ${ }^{25}$. Le lien des institutions saxonnes à l'Eglise luthérienne, de plus en plus prononcé depuis le XVlle siècle, va les caractériser et différencier des institutions de voisinages non-saxonnes.

Bien qu'anecdotiques, ces exemples illustrent néanmoins le fait que ces organisations ne puissent être comprises sans prendre en compte le réseau de relations qui les lient à des individus non-membres ou autres organisations et, successivement, aux royaumes, empires et finalement à l'État-Nation qui les entoure.

En effet, plus que le milieu spécifiquement rural ou urbain dans lesquels ces modes d'entraide s'insèrent, c'est la prise en compte de l'endroit qu'ils occupent dans la structure des réseaux qui aide à comprendre la nature, variable à travers le temps et l'espace des liens entre membres et des actions propres au fonctionnement de l'institution. 


\section{LES INSTITUTIONS DE VOISINAGES ET LEUR RÉALISATION ACTUELLE : MILIEU RURAL, MILIEU URBAIN, CONTINUITÉ(S) ET DISCONTINUITE $[S]^{26}$ ?}

\section{Dimension}

Une institution de voisinage rurale compte en moyenne une cinquante de familles, alors que les voisinages hongrois, roumains et tsiganes de Sighișoara ont au-delà de 500 familles-membres. En milieu urbain, (à Sighișoara, comme dans d'autres petites villes telles Rupea ou Medias) l'organisation en voisinages pourrait à première vue être comparée à une "assurance-mort". Prenons l'exemple du voisinage hongrois de la rue Bajor ${ }^{27}$ : le comité réuni est composé du père de voisinage, de deux vice-pères de voisinage, d'un trésorier, de trois censori qui contrôlent les entrées et les sorties financières effectuées par le trésorier, de treize "sectoristes" responsables de la circulation des informations et de la collecte d'argent dans une zone. Le voisinage compte 576 familles, chaque sectoriste est donc en contact direct avec environ 55 familles.

La totalité des membres est largement supérieure à celle observée en milieu rural. Vu le nombre élevé de membres, un père de voisinage ne connaît plus forcément chacun d'eux mais le contrôle social, loin de disparaître, est délégué au niveau du sectoriste.

Les voisinages saxons de Sighișoara, ayant vu leur effectif diminuer d'année en année et surtout depuis 1990, ont décidé de se réunir pour former une "aide générale à l'enterrement" ( $\mathrm{ABH}$ - AIIgemeine Begräbnishilfe) qui n'enlève rien à la raison d'être des différents voisinages si ce n'est que tous les Saxons de tous les voisinages doivent verser une certaine somme d'argent (5000 lei par personne en été 2001) au moment du décès du citoyen saxon de Sighișoara, inscrit dans une institution de voisinage. L'obligation de présence ainsi que de la prise en charge du transport du cercueil reste attachée aux membres appartenant au même voisinage que le défunt.

Cette réunion des différents voisinages était jugée nécessaire en vue de disposer d'un nombre assez élevé de membres pour que l'aide financière, versée au moment d'un décès soit convenable.

\footnotetext{
${ }^{26}$ Les terrains de recherche, à la base de cet article, ont été effectués en 2000 et 2001, principalement à Sighișoara, Slimnic et Mălâncrav. Sighișoara est une ville de taille moyenne, Slimnic est un village situé à proximité de la ville de Sibiu et Mălâncrav est un village isolé.

Aussi bien la ville que les deux villages connaissent l'organisation en institution de voisinage. À Slimnic, il existe dix voisinages roumains, un seul voisinage saxon - alors qu'ils ont été au nombre de quatre en 1990 - et un voisinage tsigane. Mălâncrav connaît deux voisinages roumains, un voisinage tsigane et deux des six voisinages saxons se réunissent encore en tant que tels. Pour Sighișoara, il existe dix voisinages saxons dont deux sont appelés "voisinages d'hommes" et huit "voisinages de femmes". Les Hongrois, les Roumains ainsi que les Tsiganes de Sighișoara ont créé six institutions de voisinage
}

27 Crée une première fois le 26 mai 1914, pour être relancé en 1935. 
Dans la logique des institutions de voisinage urbaines, plus il y a de membres, mieux c'est. En effet, au moment d'un décès, chaque membre de voisinage paie une certaine somme d'argent. La totalité de cette somme est versée à la famille du défunt. La somme des cotisations individuelles n'est pas calculée en fonction du prix du cercueil, elle est généralement bien supérieure à celui-ci. $\mathrm{Si}$, en milieu rural l'entraide est exprimée en argent, elle est fixée au prix du cercueil.

En milieu rural, le nombre de membres doit pouvoir répondre aux besoins pratiques de la prise en charge de l'enterrement et éventuellement de l'achat d'un cercueil. En milieu urbain, le nombre de membres devient synonyme de "richesse" et de sécurité face au coût financier important infligé par la mort d'un proche.

\section{Assurance-mort ou entraide élargie}

La prise en charge de l'enterrement constitue la fonction principale de ces différents voisinages ${ }^{28}$, à tel point qu'ils sont parfois appelés "société d'enterrement". À la question si la pratique du voisinage est d'origine saxonne, un membre d'un voisinage roumain de Slimnic, âgé de 52 ans, nous répond :

je ne sais pas, je ne sais pas. (...) Peut-être faudrait-il demander à quelqu'un de plus âgé. Moi, je ne peux le dire. A-t-elle existé avant l'arrivée des Saxons ? Je ne sais pas. (...) Je pense qu'avant que le Saxon ne soit venu, les Roumains ont bien dû avoir une manière de s'organiser, de s'ordonner. Autrement comment faisaientils ? Chacun conduisait son mort à lui, seul ? Peut-être n'était-ce pas exactement comme maintenant, néanmoins je crois que plusieurs familles, 10 à 15 familles ont bien été réunies - ça ne pouvait pas être autrement.

Notre interlocuteur lie l'existence de l'institution de voisinage directement à la prise en charge de l'enterrement. Cette association immédiate entre voisinage et enterrement est récurrente.

En milieu rural, les fonctions explicites d'entraide des différentes institutions de voisinage peuvent être plus nombreuses, mais ne le sont pas obligatoirement. Ainsi, une des deux institutions roumaines de voisinage à Mălâncrav, celle qui réunit les bastinași, les autochtones du village, ne s'active que lors d'un décès. Les membres du voisinage paient au moment de la veillée du défunt ou le jour de l'enterrement ; la somme de leurs cotisations (10 000 lei par personne en été 2001) revient au prix d'achat du cercueil (780 ooo lei), organisé pour l'occasion par le père de voisinage. Des amendes sont prévues en cas de non-paiement des cotisations. Le voisinage offre également la vaisselle, les tables et les chaises pour que la famille en deuil puisse offrir correctement la pomană (l'offrande aux morts) :

${ }^{28}$ À part l'exemple du voisinage des femmes de Sighișoara qui ont fait l'objet d'un article publié in Mihailescu (Vintila) et al., ed., op. cit. 
le voisinage chez nous est une aide plutôt pour l'enterrement, par exemple quand quelqu'un meurt, nous payons une taxe fixe. Quand quelqu'un meurt dans notre voisinage, on creuse la tombe, on l'amène (au cimetière), le cercueil est gratuit - également de notre argent. Ainsi que la vaisselle, les verres, les assiettes - chez nous se fait une pomană, après l'enterrement nous offrons un repas ; nous fournissons les tables et les chaises, le cercueil - tout, mais le reste - le morceau de poulet à table revient à la farnille. ${ }^{29}$

Selon Weber, les fonctions des institutions de voisinage se sont de plus en plus concentrées sur la prise en charge de l'enterrement après la Deuxième Guerre mondiale ${ }^{30}$. Weber ne considère que les institutions de voisinages saxonnes, mais cette même tendance est observable dans le cas des voisinages hongrois et roumains pour lesquels un éventail d'activités s'ajoutait à la prise en charge de l'enterrement ${ }^{31}$.

Cette spécialisation, présente certes de façon plus univoque en milieu urbain ne peut cependant suffire pour distinguer les voisinages en institutions rurales ou urbaines. En effet, en milieu urbain, c'est l'événement d'un décès qui déclenche l'activité proprement dite de l'organisation du voisinage. L'entraide n'y est pas basée sur la contribution financière, la présence à l'enterrement et, dans certains cas, le transport du défunt ainsi que l'excavation de la tombe qui représentent les derniers gestes de l'accompagnement en bonne et due forme du "voisin" décédé.

Au village, l'excavation de la tombe, le transport du cercueil jusque dans la tombe est affaire uniquement de l'institution de voisinage. Dans la Nachbarschaft, qui entretient un lien privilégié et direct à l'Eglise luthérienne, les membres du voisinage sont souvent responsables de sonner les cloches pour marquer les différentes étapes de l'enterrement. À chaque décès, un groupe de quatre à six membres est appelé à prendre en main la pioche et à creuser la tombe.

Cependant, au-delà de la tendance générale vers une spécialisation funéraire, des entraides et activités en réunion diverses sont présentes ici et là, dans le cadre d'organisations en voisinage. En voici deux exemples.

L'institution de voisinage tsigane de Mălâncrav prévoit d'autres formes d'entraide. Selon le trésorier, le voisinage aurait été crée en 1920. Il insiste pour dire que les institutions de voisinage sont bien plus anciennes en faisant référence explicitement aux voisinages saxons. Leur voisinage s'occupe également de l'enterrement, sur un mode similaire à celui du voisinage roumain du

\footnotetext{
29 Petit père d'un voisinage roumain à Mälâncrav

30 Weber (Georg), art. cit., p. 146.

${ }^{31}$ Voir notamment les articles de Stahl (Henri H.), "Vecinitatile din Dragus ", Sociologie Romaneasca, 1 (1), 1936 ; Schenk (Annemie), art. cit. ; Mușlea (Candid C.), " Contribuții la instituţia "vecinei" la românii brașoveni ", Studii și articole de istorie, Il, 1957 ; et Pozsony (Ferenc), art. cit.
} 
même village. Cependant, à Mălâncrav, ce sont les membres du voisinage tsigane qui s'occupent de l'entretien du cimetière. N'ayant traditionnellement pas de lien direct avec l'Eglise orthodoxe, l'institution de voisinage tsigane dispose d'un trésorier qui est assistant du prêtre orthodoxe et qui a introduit l'entretien du cimetière dans les activités de l'organisation. Par ailleurs, les membres du voisinage ont la possibilité, s'il y a assez d'argent dans la caisse, d'emprunter une certaine somme contre le paiement de $20 \%$ d'intérêts après deux semaines et sans intérêts durant cette période s'il s'agit d'un malheur affligeant une famille. "Nous sommes les plus pauvres, nous n'avons personne pour nous aider [il fait référence aux Saxons qui reçoivent de l'aide de ceux qui sont émigrés en Allemagne] mais nous nous soutenons l'un (à côté de) l'autre, comme nous le pouvons."

La taxe d'inscription est bien plus élevée (180 o0o lei par famille en été 2001) que dans d'autres voisinages et il en va de même pour les amendes ; l'argent réuni de cette manière fonctionne en véritable assurance-malheur. Les amendes sont non seulement plus élevées, mais également multiples. Il y a obligation de présence d'un membre de chaque famille à la veillée du mort, à l'enterrement, aux réunions officielles comme à la fête du voisinage, d'être ponctuel aux différentes occasions, de ne pas être ivre, etc. Si un membre ne respecte pas ces règles inscrites dans les statuts du voisinage, il est exclu de celui-ci. Les règles énoncées ne correspondent pas seulement à des normes théoriques, les exclusions de membres peuvent être vérifiées dans les procès verbaux des réunions de voisinage dans lesquels tous les détails de la procédure sont minutieusement notés.

Si tu ne nous accompagnes pas, et si ça ne te convient pas, alors nous te mettons à la porte, sans aucun commentaire. Ça c'est le voisinage! Pour le meilleur et pour le pire. Quand il est question de travail, tu viens travailler ; pour la fête nous ne t'avons pas demandé un sou, mais si cela ne te convient pas, tu ne restes plus dans nos rangs. Ca c'est la loi.

À Slimnic, le maire contactait tous les pères de voisinages roumains et saxons jusqu'en 1962 pour les Roumains et 1945 pour les Saxons) ${ }^{32}$. Tous ceux qui disposaient de bétail étaient obligés d'aller couper du bois dans la forêt communale qui servait à l'entretien de l'infrastructure et des bâtiments publics du village. Les responsables de travaux s'adressaient aux pères de voisinage et les informaient du nombre d'hommes et de charrettes dont ils avaient besoin pour abattre les arbres signés au préalable. Des anciens ponts étaient rénovés et des nouveaux ponts construits à l'aide de la coopération des pères de voisi-

${ }^{32}$ Le trèsorier d'un voisinage roumain, lui-mème maire retraité de Slimnic évoque ici l'année (1962) quí achève le processus de collectivisation des terres ainsi que la fin de la guerre, l'expropriation des maisons et des terres des Saxons; la plupart des maisons ont été rendues en 1956. 
nage qui envoyaient pour chaque jour de travail un certain nombre d'hommes sur les chantiers. Au printemps, c'était également via les institutions de voisinage qu'étaient remis à neuf les chemins qui menaient aux champs et les murs de l'école de Slimnic furent restaurés à travers la coopération entre différents voisinages et la mairie.

Comme nous avons pu le montrer, l'entraide élargie a continué d'exister après la Deuxième Guerre mondiale, pour s'exprimer de diverses manières aujourd'hui encore. Cependant, ceci n'est pas le cas pour chaque voisinage et, alors que tout voisinage était concerné par une multitude de fonctions, telle la sécurité, l'entretien du village, les enterrements, le carnaval, la construction de maisons, l'institution du voisinage est actuellement d'abord et avant tout définie par la prise en charge des enterrements.

Toujours selon Weber, l'entraide familiale, après la Deuxième Guerre, serait censée venir entièrement de la part de la parenté et non plus de la Nachbarschaft. Si la parenté fait défaut, c'est la communauté religieuse évangélique qui vient en aide. La Nachbarschaft n'en viendrait qu'à revêtir face à la famille nucléaire qu'une fonction d'allègement en ce qu'elle activerait la conscience commune d'un rattachement à une communauté. L'aide émanant du voisinage ne serait plus constitutive pour le groupe et la demande d'aide au voisin deviendrait problématique et suspecte, suite à la méconnaissance de la situation économique réelle de l'autre33.

Le régime socialiste, à travers la collectivisation des terres et le travail dans les fermes collectives et d'État, l'augmentation de la mobilité professionnelle, a certainement influencé le rapport à la propriété, au travail quotidien et finalement à l'entraide, la mort lui aurait-elle échappé ?

Au-delà des approches rituelles orthodoxes, protestantes, catholiques et autres, différentes par rapport à la mort, celle-ci n'est en aucune façon tabouisée. Et finalement, c'est la mort qui est au centre des activités et qui réactive les lieux d'interaction des membres des voisinages. Pour un Roumain, l'enterrement des Saxons, n'offrant pas la pomană, est souvent jugé comme très et trop sobre. Par contre, en Allemagne, nous avons souvent pu entendre l'étonnement que suscitait l' "hypertrophie" de la mort chez les Saxons. Ce n'est certes pas par nécessité arbitraire que l'institution se crée et se recrée autour de l'événement de la mort pour en faire sa spécialité proprement dite. C'est au moment d'un décès que les divers réseaux d'entraide s'activent et l'institution du voisinage, en tant que réseau parmi d'autres (parenté, amis, collègues, ...) et se superposant à d'autres, contribue de manière formelle à cette prise en charge collective d'une mort qui, en termes relationnels de réseaux, n'est autre que collective à son tour. Le sens de l'action, en passant par une recherche de l'avoir, revient en définitive à une logique de l'être face à la mort. 


\section{Discours normatif et amendes - quantification de l'entraide}

En milieu urbain, le père du voisinage est également le premier informé lors du décès d'un des membres, il se charge de communiquer la nouvelle au trésorier qui peut alors retirer l'argent en réserve à la banque pour verser, le deuxième jour après le décès, une aide à la famille du défunt 34 . Les membres du voisinage paient le transport du défunt, pris en charge par des employés communaux, de la maison à la chapelle et de la chapelle à la tombe, le troisième jour, au moment de l'enterrement. Avant 1998, le sectoriste responsable devait organiser des membres de son secteur pour transporter le défunt 35 , Mais comme le constate le père de voisinage : " c'est ça l'économie de marché, tout doit être payé, tout le monde le dit. En vain tu leur dis que ceci est une association d'entraide" .

L'institution appelée institution de "voisinage" et suscitant une image idéale et idéelle de la communauté et des liens communautaires "traditionnels" entre paysans, aurait-elle ici passé le cap de la Gemeinschaft vers la Gesellschaft?

La quantification et la fixation de l'entraide, permet-elle de parler d'une rupture véritable entre logique propre au don et logique de contrat ?

Les sectoristes organisent entre eux une réunion au moins une fois par an et chacun y rapporte qui des membres n'est pas discipliné en n'étant, par exemple, jamais présent aux enterrements. Sur base de leur concertation ils rédigent un rapport dont on tiendra compte lors de la réunion annuelle de l'institution de voisinage, obligatoire, sous peine d'amendes pour chaque membre. Ce sont également les sectoristes qui après un enterrement, font la collecte de l'argent dû par chaque famille-membre ${ }^{36}$ (7000 lei en été 2001). Si un membre ne paie pas "trois morts" il est exclu du voisinage. Les amendes, bien plus élevées du côté roumain que hongrois, nous semblent être plus symboliques que réelles. Le père du voisinage hongrois disait à ce propos :

à la non-participation à l'enterrement il faut payer une amende, de 1 ooo lei parce nous avons constaté qu'en vain nous exigeons 10 ooo lei qui ne seront pas payés.

\footnotetext{
34 L'aide consiste en une somme d'argent (4 200000 lei en été 2001) et en accessoires nécessaires à l'enterrement : une table sur laquelle on pose le mort, des tissus noirs pour couvrir cette table et encore une petite table avec une couverture noire sur mesure et un drapeau qui sera accroché à la porte de la maison du défunt.

35 Dans le voisinage nommé numéro I, Cornești-Tâmavă et réunissant 446 membres, ce sont les hommesmembres du voisinage qui creusent la tombe pour leur membre défunt et ils sont payés par le fond du voisinage pour ce service.

${ }^{36} \mathrm{La}$ cotisation des familles dans le voisinage roumain sus-indiqué est de 15000 lei, et les taxes d'inscription, variant selon l'âge du futur membre, représentent également le double des cotisations dans le voisinage hongrois dans lequel les membres paient par famille alors qu'on paie par personne dans le voisinage roumain. En effet, les frais d'inscription représentent, en été 2001 , entre 30 et 40 ans 50000 lei, entre 40 et 50 ans 70000 lei et entre 50 et 60 ans 100 ooo lei.
} 
Chez les Saxons, le sentiment du devoir est plus élevé, et quand le Saxon ne s'est pas présenté volontairement [à l'enterrement], il dit : "s'il vous plaît voici mon amende!"

Pourtant, même symbolique et non contraignante, l'amende en tant que telle existe dans toutes les institutions de voisinage. Son existence vient souligner le discours normatif qui, exprimé à la réunion générale, rappelle les obligations des membres par rapport au voisinage en tant qu'organisation d'entraide. L'amende symbolise l'exigence des devoirs de la part de chaque membre.

À la réunion annuelle, les différents membres du conseil présentent leur rapport et le père de voisinage mentionne les décès de l'année écoulée, juge le comportement du "collectif" lors de ces enterrements, cite les exemples négatifs mais aussi positifs et loue ceux qui se sont distingués.

Le trésorier d'un voisinage roumain de Sighişoara définit son organisation et son rôle en tant que trésorier en ces mots : " c'est une association sans but lucratif, quand l'homme meurt, j'y vais et je collecte l'argent ".

À cette entraide qui fait appel au calcul et aux aspects très concrets d'ordre financier, est toujours lié un discours normatif et moralisateur. Les pères de voisinage valorisent ou dévalorisent les présences, les absences et les engagements de ses membres, l'entraide en tant que telle est explicitement articulée. Le trésorier du voisinage numéro I qualifie la nature des devoirs et des droits énoncés dans les statuts de voisinage comme étant des " normes de l'homme civilisé $"$. La quantification de l'aide et des obligations en unité monétaire n'est pas liée, comme on pourrait se l'imaginer, au contexte urbain. En effet, elle fait intégralement partie du patron de l'institution de voisinage, qu'elle soit rurale ou urbaine. Cependant, en milieu rural, l'argent se substitue rarement aux différents actes d'entraide proposés.

Max Hedley désamorce la dichotomie entre logique du don et logique de contrat :

it can be suggested that the new ways are no more than a precise formalization of a transactional element that, while de-emphasized, was always present. The difference in the past was not the absence of calculation in these relationships between households, but the diffuseness of its structure, diffuseness which was present with respect to what should be returned and the time at which it should be repaid. 37

Alors que l'évaluation, en termes monétaires, des devoirs manqués des membres est présente dans toute institution de voisinage, l'entraide ellemême tend à être remplacée en partie par une somme d'argent effective dans le cas des institutions de voisinages urbaines. En d'autres mots, ce n'est plus le 
cercueil qui est fourni par l'institution de voisinage, mais une somme d'argent dont le montant dépend du nombre de membres inscrits. La famille du défunt est libre d'en disposer comme bon lui semble.

Selon Godbout, " le don fuit le calcul, ce qui l'oppose autant au principe public d'égalité qu'au principe marchand de l'équivalence $~^{3^{8}}$. Le calcul dans notre cas, n'est pas périphérique mais au centre de l'entraide ; seulement cette entraide, non spontanée mais volontaire n'a pas pour fin le profit économique ou le pouvoir. L'objectif de cette entraide est la recherche d'un sentiment de sécurité procuré par l'insertion dans un réseau social qui garantit une aide au moment d'un décès. Il ne s'agit pas d'individus qui s'engagent dans une transaction marchande, mais de "ménages" qui s'insèrent dans un réseau de liens dont ils ne s'isolent pas. Il nous semble difficile de mettre le don du côté des institutions de voisinages ruraux et l'absence du don du côté des institutions urbaines. Tous les voisinages opèrent avec le calcul et l'argent ainsi qu'avec la garantie de la réciprocité, mais celle-ci n'est pas immédiate ni équivalente à ce qu'un ménage a pu donner au cours des années.

\section{L'appartenance ethnique et les appartenances multiples Critère terRItorial relativisé}

Alors que le principe territorial était le critère par excellence pour déterminer l'appartenance à une institution de voisinage et notamment à la Nachbarschaft, l'importance exclusive de ce critère tend à diminuer pour être remplacé par des liens de parenté et d'affinités diverses.

En effet, en milieu rural, le critère territorial voit sa performance diminuée même pour les quelques voisinages saxons qui existent et pour qui la proximité physique des fermes était l'élément constitutif par excellence de tout "voisinage".

En général, ce ne sont plus les voisins proprement dits qui forment un voisinage mais bien plus des parents, des amis ou des "nouveaux-venus" au village dont les habitations peuvent être dispersées dans le village entier.

C'est en milieu urbain que le lien au territoire joue un rôle plus prononcé en ce qui concerne l'appartenance à une institution de voisinage. Certes, le critère de proximité des habitations n'est pas le seul à être pris en considération, il tend néanmoins à se superposer, du moins en partie, au critère d'appartenance ethnique. Le voisinage roumain de Cornești-Tâmavă compte parmi ses membres également des familles hongroises et tsiganes. Ces familles sont membres parce qu'elles habitent les quartiers relevant d'un responsable sectoriste d'un voisinage donné. Rien n'empêche ces familles de s'inscrire parallèlement au voisinage hongrois et tsigane respectif. À part le quartier de la gare, qui connait une concentration d'une population plus particulièrement tsi- 
gane, la ville ne peut plus être découpée en "quartiers à prédominance ethnique" et elle est habitée par des Tsiganes, Roumains, Hongrois et Saxons tous confondus.

La rue Galtberg à Sighișoara est traversée par deux institutions de voisinages, l'une appelée "saxonne" et l'autre "hongroise". Cependant, les Saxons s'étant référé à leur Rauchprinzip ${ }^{39}$, les habitants du haut de la rue Galtberg ne devaient pas appartenir à la Nachbarschaft Galtberg. Ces habitants saxons du haut de la rue ont dû s'inscrire, non sans polémiques, dans l'institution de voisinage hongroise.

Cependant, si le critère territorial est pris en compte, il n'est pas exclusif. En effet, les statuts du voisinage Bajor prévoient que les individus déménageant dans une autre ville aient la possibilité de rester membres à vie, moyennant paiement à travers des membres de sa famille, des amis ou des voisins restés à Sighișoara. Cependant, si quelqu'un quitte le pays, il restera membre pendant cinq ans, toujours en assurant les cotisations, et s'il ne revient pas s'installer au pays après cette période, il perd le statut de membre.

Le choix d'une institution de voisinage est lié désormais à l'appartenance ethnique, au lieu de résidence éventuellement, ainsi qu'à la confession. À cela peuvent s'ajouter d'autres considérations, telles que le milieu social (il existe des voisinages nommés "d'élite" ou "d'intellectuels") et le lieu de provenance.

À Sighișoara, par exemple, s'est crée un voisinage hongrois dont les membres proviennent majoritairement du village Jakodu ; l'institution de voisinage en question s'appelle "voisinage de Jakodu". Le père de voisinage Bajor nous dit que certains de ses membres sont également membres du voisinage Jakodu.

En milieu urbain, les époux d'un mariage mixte s'inscrivent souvent dans chacune des institutions de voisinage respective.

\section{LA DOUBLE INSCRIPTION}

La double inscription dans le voisinage, suite aux mariages mixtes ou non, n'est possible qu'en ville. En effet, à Sighișoara il n'est pas rare que les habitants qui peuvent se le permettre financièrement, s'inscrivent dans deux institutions de voisinage. Une femme saxonne de Sighișoara expliquait que les voisinages roumains versent à leurs membres une somme bien plus importante en cas de décès, grâce à leur nombre élevé et que dès lors "ça vaut la peine d'y mourir ". Le père de voisinage d'une institution hongroise nous informait que les Hongrois ont également crée des institutions de voisinages sur base confessionnelle et que lui, à côté de "son " voisinage (de confessions mixte), s'était inscrit au voisinage hongrois catholique. Il dit ne pas savoir 
comment fonctionne l'autre voisinage, il ne s'y rend que pour payer les cotisations et les amendes.

Le but visé de la double inscription n'est cependant pas motivé par une maximisation de profits escomptée d'un parent décédé mais dénote d'une recherche d'un meilleur départ, où devrait-on dire d'un "bon passage" d'un être proche vers l'autre monde.

Cette double inscription, pratiquée de façon récurrente en milieu urbain, est absente en milieu rural. Nous n'avons jamais pu y observer l'inscription d'un individu dans deux voisinages. La valorisation du groupe et le sentiment d'appartenance qui en découle semblent exclure la possibilité d'élargir son statut de membre à deux institutions de voisinage.

Pahl propose la notion de densité de texture de rôle pour rendre compte d'une situation dans laquelle les rôles des individus sont si bien définis qu'il leur devient difficile d'en revêtir plusieurs à la fois. Dans un réseau au sein duquel les liens entre membres donnés sont empruntés et réempruntés dans un éventail à des contextes différents et qu'on pourrait alors qualifier de " multiple-way relationships $" 4^{\circ}$, la densité du rôle est telle qu'elle est susceptible de produire un fort sentiment d'attachement au réseau donné41.

Alors que le village traditionnel répond à une définition de forte densité de texture de rôle, la distinction entre milieu urbain et rural ne peut pas, a priori, être pertinente par rapport à la nature des liens qui définissent autant de réseaux, qu'ils soient urbains ou ruraux.

" Donner, recevoir et rendre " est une spirale qui, en milieu rural, s'insère dans un réseau à forte densité de texture de rôle alors qu'en milieu urbain, elle peut s'inscrire dans deux ou plusieurs réseaux à faible densité des relations respectives. En ville, un membre inscrit dans deux voisinages donne plus mais reçoit également plus et l'entraide peut se multiplier puisque son unité d'expression explicite est l'unité monétaire. Dans le village, deux cercueils ou deux tombes pour un défunt ne riment à rien. Pourtant, même en ville, la présence des membres à l'enterrement, vivement souhaitée, ne peut, elle, pas non plus être multipliée.

La double inscription se révèle être la seule véritable rupture entre voisinages urbains et voisinages ruraux. La qualité des liens qui lient les individus dans le réseau est à la base de cette rupture. Au-delà d'une entraide en large mesure (mais non exclusivement) exprimée en unités monétaires, c'est également la valeur de l'appartenance ethnique en tant que critère d'ouverture et d'accessibilité qui diffère entre voisinages urbains et ruraux. 


\section{APPARTENANCE ETHNIQUE}

Nous avons jusque là utilisé le terme "ethnie" 42 , sans y apporter aucune critique. Tout en étant d'accord avec la sévère remise en question de la pertinence à priori explicative d'un tel concept, la catégorisation ethnique, construite, est néanmoins efficace dans le cas présent et ne se réduit en aucune façon à une création du chercheur. Elle joue un rôle important dans les réseaux de relations entre individus ainsi que dans le discours que ceux-ci produisent sur eux-mêmes.

Les institutions de voisinage recrutent leurs membres en fonction de leur appartenance ethnique. Le critère "ethnique" n'est nulle part requis explicitement dans les statuts - exception, faite dans les années 1930, des prescriptions administratives saxonnes, sus-mentionnées - mais il s'agit d'un critère nécessaire et suffisant sur base duquel un voisinage peut être formé. Sans être exigée, cette appartenance ethniquement homogène se dessine néanmoins dans les faits et dans la dénomination des institutions de voisinage. Cependant il existe des dérogations à ce principe de fait.

Dans le cas de mariages mixtes, il est possible que l'un ou l'autre "étranger" fasse partie d'une institution de voisinage. Les partenaires "étrangers" parlant la langue dudit voisinage, en font partie en tant que membre entier. Ainsi, la Nachbarschaft numéro 5 de Malmkrog est composée d'hommes saxons qui pour la plupart sont mariés à des femmes roumaines, tsiganes ou hongroises. Il arrive que le partenaire "étranger" fasse partie de l'institution de voisinage de son époux ou de son épouse ainsi que de celle de son groupe ethnique.

L'appartenance ethnique en milieu rural est bien plus un "enjeu" pour un individu donné que cela ne semble être le cas en milieu urbain. L'histoire de l'individu est rattachée d'office à l'histoire de son groupe. L'exemple de Mălâncrav est parlant à cet égard. Les habitants de ce village, paysans, artisans avant la Deuxième Guerre ont travaillé ensuite en majorité dans la ferme collective près du village et retournent à une économie d'autarcie de petits paysans après 1990. L'Histoire avec un grand $\mathrm{H}$ a traversé le village et s'est traduite localement en histoires familiales, d'amitiés et de voisinages. Ces étapes sont plus ou moins remémorisées, plus ou moins mises en évidence dans le discours partagé à Mălâncrav. Les habitants connaissent les Saxons qui se sont engagés dans la Wehrmacht-SS 43 et ils connaissent leurs descendants actuels, tous savent qui fut déporté après la guerre et quelles maisons saxonnes "vides"

$4^{2}$ Voir notamment l'ouvrage récapitulatif sur le concept d'ethnie : Banks (Marcus), Ethnicity : Anthropological Constructions, London : Routledge, 1996.

43 Suite au contrat du 12 mai 1943 entre la Roumanie et l'Allemagne, tous les citoyens roumains de nationalité allemande sont libres de s'engager dans la Wehrmacht-SS allemande, ce qu'ils firent en grande majorité. 
ont changé de propriétaires par la force ; les descendants de ces nouveaux propriétaires, roumains et tsiganes, cohabitants avec les Saxons dans les maisons de ceux-ci, vivent en partie toujours au village. En 1990, ce sont les habitants saxons qui ont eu la possibilité d'émigrer en grand nombre en Allemagne pour y devenir citoyens allemands 44 .

Les réseaux de relations entre groupes et individus dans cette collectivité sont fortement teintés "ethniquement" et le méta-discours des habitants vient souligner cette différenciation sur base ethnique. Cependant, il nous semble important de procéder à une inversion de causalité. En effet, au lieu de déduire le sens d'une action, qu'elle soit individuelle ou collective, sur base de l'appartenance ethnique, il nous semble plus judicieux de comprendre la référence au groupe ethnique à travers les actions et les représentations qui ont pu le construire et le faire perdurer.

À Mălâncrav, le Tsigane qui fait partie du voisinage roumain coloniste, y adhère moins en tant que villageois, habitant de Mălâncrav que par volonté de se rattacher au groupe des Roumains colonistes et non plus au voisinage tsigane. Par contre, un Tsigane peut être jugé recevable de par ses bonnes qualités qui font oublier son statut de "Tsigane" pour accéder à celui de Roumain. S'il est désigné comme om cum să cade (un homme correct), il inspire confiance et peut changer de groupe. Le changement de statut s'accompagne d'une renonciation à l'ancienne appartenance, la double appartenance, du moins formelle, ne se pratique pas. Lorsqu'il reste dans le voisinage tsigane, ses justifications sont explicitement liées à des considérations d'appartenance de groupe. Le trésorier du voisinage tsigane de Mălâncrav nous dit : " il y a des Tsiganes qui sont chez les Roumains [dans le voisinage roumain]. J'ai été invité en personne par les Roumains, mais je n'ai pas voulu quitter. (...) Moi, rien ne me dérange et personne ne peut me porter aux nues ; car, si je suis Tsigane, je suis Tsigane, mais le destin humain, il faut que tu le respectes [conserves] ".

De même, un Saxon à qui nous avons demandé s'il pouvait s'inscrire dans un voisinage roumain, toujours à Mălâncrav, s'exclame : " mais, je ne veux pas, je pourrais peut-être, je ne sais pas comment ça fonctionne chez les Roumains, mais (...) ils ont aussi d'autres coutumes, et puis alors autant de Roumains rassemblés et seulement un Saxon, alors on est euh (...) ils triompheraient et diraient : oh, le Saxon est venu chez nous!"

L'appartenance au voisinage se pose ici dans des termes exclusifs, alors que l'appartenance ethnique n'est en rien évacuée dans le contexte urbain, elle ne se subordonne pas sur un mode univoque à l'appartenance au voisinage.

En approchant l'institution de voisinage en tant que réseau d'entraide s'insérant dans d'autres réseaux sociaux, il est possible de mettre en évidence,

44 Rien que pour l'année 1990, au-delà de 111000 "Allemands" quittent la Roumanie (Gündisch (Konrad), op. cit., p. 246). 
en contexte urbain, des liens à la fois "urbains", c'est-à-dire tendant vers l'anonymat, et de nature plus indirecte que directe ainsi que des liens communautaires ou locaux. L'individu en tant qu'élément d'une multitude de réseaux de relations sociales, n'est pas rattaché exclusivement à un nœud, cluster du réseau. Les liens entre individus et collectivités en milieu rural, isolés tant spatialement que socialement, réactivent régulièrement les mèmes connections et ce à des occasions et pour des besoins divers. "As a result, "institutionally complete" ethnic groups - supplying a broad range of services to members tend to retain comparatively high proportions of their members' informal contacts. " 45

Le réseau de l'entraide formelle est ici largement superposé au réseau ethnique, alors que les contacts et les entraides informels quotidiens ne respectent pas forcément les frontières construites ethniquement.

Ce n'est pas la localisation en milieu rural en tant que telle qui peut expliquer la nature de l'entraide. Slimnic en tant que village à proximité d'une ville, est habité "d'étrangers" au village, de travailleurs et d'élèves ou d'étudiants qui quittent quotidiennement le village pour la ville ainsi que de paysans et d'individus vivant la majorité de leur vie au village. En effet, tous ne font pas partie d'une institution de voisinage et il y a des réseaux d'entraides, familiaux, religieux et autres qui, soit se superposent au voisinage, soit l'excluent.

La double inscription ou l'appartenance multiple qui permet de distinguer les pratiques urbaines et rurales met en évidence une discontinuité nette entre l'institution de voisinage urbaine et rurale. Cette discontinuité relève de l'univocité ou au contraire de la multiplicité de l'appartenance aux groupes d'un côté ainsi que de l'aide en termes surtout pécuniaires ou en nature de l'autre côté. En effet, en milieu rural, l'argent est utilisé pour juger et fixer la valeur des devoirs des membres à travers les amendes ainsi que pour fournir le cercueil. Par contre, en milieu urbain, l'argent devient le plus souvent en tant que tel l'objet de l'entraide.

Ce n'est pas parce que l'on se trouve dans une ville plutôt qu'au village que la double inscription devient en soi possible. C'est la multiplicité des réseaux d'appartenance ainsi que l'accent mis sur une mëme unité de valeur pour exprimer l'entraide qui rendent possible cette double inscription, récurrente ou absente, dans les institutions de voisinages.

Le critère d'appartenance au voisinage est bien sür le critère ethnique mais également celui d'affinités et de parenté ainsi que de provenance ou de "statut social". Ni ouverte de fait à tout un chacun, ni fermée hermétiquement, toute institution de voisinage, urbaine comme rurale, est d'accord pour pren- 
dre en charge l'enterrement d'un étranger, non-membre du voisinage, si la famille de celui-ci paie les frais dont le montant est prévu dans les statuts et qui sont élevés eu égard à la non-participation de cet étranger aux activités et aux devoirs dus en tant que membre sur une période qui peut représenter toute une vie d'adulte.

\section{CONCLUSIONS}

L'institution du voisinage, en tant qu'institution transylvaine, traduit bien la construction efficace de l'ethnicité et la réification des groupes ethniques dans cette région du monde. Ces réseaux "ethniques" ont été définis et se sont définis en faisant appel à des référents variables et en bénéficiant de statuts divers au cours des siècles. Cependant, ces groupes appelés aujourd'hui "ethniques" se sont construits dans la longue durée et se reconstruisent quotidiennement en relation les uns aux autres, aussi bien de l'intérieur que de l'extérieur. À ce jour, l'appartenance ethnique, en tant que critère constructif de groupe continue à être performant.

Plutôt que de partir analytiquement de groupes discrets, tant en ce qui concerne les institutions de voisinage que les groupes ethniques, nous proposons de les aborder en tant que réseaux qui, toujours ouverts, présentent l'avantage de la prise en considération du changement continuel (variabilité intra et inter ethnique des institutions de voisinage), des relations entre exigences collectives et individuelles (par exemple les processus de prise de décisions), du lien entre niveau micro et macro et de la structuration de l'action sociale à travers des modèles de relations supra-individuelles (insertion des voisinages dans d'autres réseaux). " One can never simply appeal to such attributes as class membership or class consciousness, political party affiliation, age, gender, social status, religious beliefs, ethnicity, sexual orientation, psychological predispositions, and so on, in order to explain why people behave the way they do. $" 46$

Il serait intéressant d'analyser de plus près le rôle que peut jouer l'institution de voisinage en tant que complément formel et élargi des réseaux d'entraide de l'économie informelle tel notamment la maisnie 47 , mise en évidence par Vintila Mihailescu : " c'est au niveau de la maisnie et non pas de l'individu

${ }^{46}$ Emirbayer (Mustapha), Goodwin (Jeff), " Network Analysis, Culture, and the Problem of Agency ", American Journal of Sociology, 99 (6), 1994, pp. 1414-1415.

47 Terme proposé par Paul Stahl pour traduire le mot roumain gospodarie (household). 
que se joue le jeu des "exigences individuelles et collectives" et que vont s'articuler, plus tard, les caractéristiques rurales et urbaines et agraires et industrielles $n 48$.

Alors que les institutions de voisinage se préoccupent aujourd'hui plus particulièrement de la prise en charge de l'enterrement et ne fonctionnent que sporadiquement comme lieux d'échanges communautaires variés dans la logique du don et du contre-don à l'intérieur d'une communauté donnée, elles constituent plutôt des réseaux d'entraide spécifique qui s'adaptent de manière étonnante aux besoins donnés d'une collectivité. Ne devant répondre à des organes hiérarchiques supérieurs, les institutions de voisinage fonctionnent en tant qu'organisations d'entraide indépendantes et, de ce fait, flexibles. Souvent, les membres d'un voisinage ne sont pas au courant de ce qui se passe dans un autre voisinage du même village. Les statuts et les conventions non formalisées varient d'un voisinage à un autre et toute institution de voisinage est activement façonnée par ses membres qui, au moment des réunions annuelles, sont libres de proposer des changements.

Cette entraide, comblerait-elle un vide, suite à une démission de l'État par rapport au domaine social après le communisme? Godbout nous dit que le don

n'est pas le complément du marché ou du plan, mais celui de l'économie et de l'État. (...) Là, (...), où le marché et l'État ne parviennent pas ou plus à s'organiser, subsiste encore, ultime garde- fou, le réseau des relations interpersonnelles cimenté par le don et l'entraide qui, seul, permet de survivre dans un monde de folie. Le don ? Ce qui reste quand on a tout oublié, et avant qu'on ait tout appris ?49

Cette volonté d'entraide a fait preuve de flexibilité et de capacité d'adaptation à travers toutes ces époques. Le sens que ses membres ont pu lui attribuer et qui probablement varie non seulement d'individu à individu mais encore à travers le temps est pourtant toujours coloré d'une valorisation morale projetée sur l'institution en tant que telle.

Ce serait à l'intérieur d'institutions que se transmettent le mieux les systèmes de valeurs, de nature conservatrice, en décalage avec la réalité vécue. L'institution de voisinage se prête à l'analyse de la dissonance qui sépare les systèmes de valeur de la réalité, qui les entourent. Selon Paul Veyne :

une institution est une situation où les gens, à partir de mobiles qui ne sont pas nécessairement idéalistes - faire carrière, ne pas se brouiller avec le milieu, ne pas vivre en état de déchirement -, sont amenés à remplir des fins idéales, aussi scrupuleusement qu'ils s'intéressaient à ces fins par goût personnel; on voit donc que les valeurs qui sont à l'origine et à la fin de l'institution ne sont pas celles qui la

\footnotetext{
${ }^{48}$ Mihailescu (Vinta), " Du village à la ville et retour. La maisnie diffuse en Roumanie n, Bulletin of the Ethnographical lnstitute, 44, 1995.
}

49 Godbout (Jacques T.), op. cit., p. 26. 
font durer. D'où une tension perpétuelle entre le désintéressement que supposent les fins de l'institution et l'égoïsme naturel de ses membres ; (...) les motifs idéologiques sont les vrais quand il y a institution : chaque époque fait et vante ce qui la conditionne et la passionne (...). La clé de cette évolution est une réaction humaine si puissante qu'elle ressemble à un calcul de l'instinct de conservation : faire de nécessité vertu, changer ses maximes plutōt que la fortune, prendre les attitudes de son rôle. Puis au fur et à mesure que les générations se succèdent, il leur est plus facile de reprendre des modèles qui ont fait leurs preuves que de réinventer le monde. 50

La transmission de l'institution en tant qu'institution d'origine saxonne n'est certes pas innocente par rapport à l'image positive et le prestige dont jouissent en général les Saxons en Transylvanie, dans un contexte dans lequel l'articulation des frontières de groupes ethniques est si présente.

Toujours appelée Nachbarschaft et toujours présidée par un "père" de voisinage, cette institution de voisinage transylvaine, sans forcément tenir compte de "voisins" et organisée bien souvent plutôt par un président que par un père métaphorique, se prête à la réinvention de réponses à fournir face aux besoins d'entraide. Ne trouvant sa place dans la dichotomie de la Gemeinschaft et de la Gesellschaft, elle s'insère dans une dynamique d'adaptation qui ne propose ni un renouvellement radical ni une répétition à l'identique. Il semble difficile également d'affirmer une évolution diachronique sur l'échelle liant la communauté d'un côté à la société de l'autre.

Bruno Latour parle d'hybrides :

les deux extrëmes, le local et le global, sont beaucoup moins intéressants que les agencements intermédiaires nommés ici réseaux ; (...) les mots local et global offrent des points de vue sur des réseaux qui ne sont par nature ni locaux ni globaux, mais qui sont plus ou moins longs et plus ou moins connectés. (...) Au milieu, où rien r'est supposé se passer, il y a presque tout.51

La discontinuité imposée entre passé et présent, monde traditionnel et modernité, ruralité et urbanité sont des produits de nos catégories d'analyses et de leur réification. Il y a changement, mais il ne trouve certainement pas sa traduction sur une échelle évolutionniste entre deux dichotomies conceptuelles. Une compréhension de l'entraide n'est qu'une particule dans cette perspective. Elle est importante non seulement grâce au rôle qu'elle a joué dans nos communautés rurales mais également parce qu'elle occupe une place centrale dans cet imaginaire qui consomme la rupture entre le "bon paysan" d'antan et "l'individu égoïste" moderne. 